\title{
Isoflavone Content and Anti-acetylcholinesterase Activity in Commercial Douchi (a Traditional Chinese Salt-fermented Soybean Food)
}

\author{
Yaqiong LIU $^{1 \dagger}$, Lijun WANG ${ }^{1 \dagger}$, Yongqiang CHENG ${ }^{1}$, Masayoshi SAITO ${ }^{2}$, \\ Kohji YAMAKI ${ }^{3,4}$, Zhihong QIAO ${ }^{1}$ and Lite $\mathrm{LI}^{1 *}$ \\ ${ }^{1}$ College of Food Science and Nutritional Engineering, China Agricultural University \\ (Beijing 100083, P R China) \\ ${ }^{2}$ Research Planning and Coordination Division, Japan International Research Center for Agricultural \\ Sciences (Tsukuba, Ibaraki 305-8686, Japan) \\ ${ }^{3}$ Post-harvest Science and Technology Division, Japan International Research Center for Agricultural \\ Sciences (Tsukuba, Ibaraki 305-8686, Japan) \\ $\dagger$ These authors contributed equally to this work.
}

\begin{abstract}
The concentration and distribution of isoflavones in 19 representative commercial douchi products and their acetylcholinesterase (AChE) inhibitory activity were investigated in this study. Isoflavone aglycones are the predominant isoflavone forms in Chinese commercial douchi samples. The total content of isoflavones in douchi extracts were observed from 24 to $1,471 \mu \mathrm{g} / \mathrm{g}$ (dry matter). Results indicated that Aspergillus-type douchi had more isoflavone aglycones content than that of Mucor-type and Bacillus-type douchi. Nineteen samples also showed various extents of AChE inhibitory activity. The $\mathrm{IC}_{50}$ value of AChE inhibitory activities of douchi extracts ranged from 0.040 to $2.319 \mathrm{mg} / \mathrm{mL}$. Aspergillus-type douchi extracts exhibited significantly higher AChE inhibitory activity than that of Mucor-type and Bacillus-type douchi. Some brands of douchi which have low contents of isoflavone aglycones showed much lower inhibitory activity. However, high inhibitory activities did not mean high isoflavone content, maybe some other substances contributed to the inhibition which should be further studied.
\end{abstract}

Discipline: Functional food

Additional key words: Alzheimer's disease, fermented food

\section{Introduction}

Traditional fermented soybean foods have been consumed by Asians for a significant part of their long history. There are various fermented soybean products such as douchi, sufu, natto, miso, soy sauce, and tempeh in Asian countries which play an important role in local diets. Douchi is a traditional salt-fermented soybean food which has been consumed for more than two thousand years in China ${ }^{1}$. Most douchi products are produced by a similar principle; the schematic diagram for production of douchi is shown in Fig. 1. Production of douchi involves four major steps: 1) soaking soybean; 2) steaming soybean;
3) inoculation; and 4) ripening. According to the microorganism used, Chinese douchi can be divided into three types: Aspergillus-type, Mucor-type and Bacteria-type.

Recently, many physiological properties have been found in Chinese douchi, which include anti-oxidative activity $^{26,30}$, thrombolytic effects ${ }^{20}$, anti-diabetic properties ${ }^{2}$, and anti-hypertensive effects ${ }^{35}$. In many respects, isoflavones are one of the most important compounds attributed to those healthful effects. Many investigations were made previously on a wide variety of soybeans, non-fermented soybean foods and fermented soybean food to evaluate the potential of isoflavones as a dietary anti-carcinogen by some researchers ${ }^{27,28}$. However, there is little information available regarding isoflavones content in Chinese

\footnotetext{
Present address:

${ }^{4}$ Food Function Division, National Food Research Institute, National Agriculture and Food Research Organization

(Tsukuba, Ibaraki 305-8642, Japan)

*Corresponding author: e-mail 11t@cau.edu.cn

Received 11 August 2008; accepted 4 January 2009.
} 


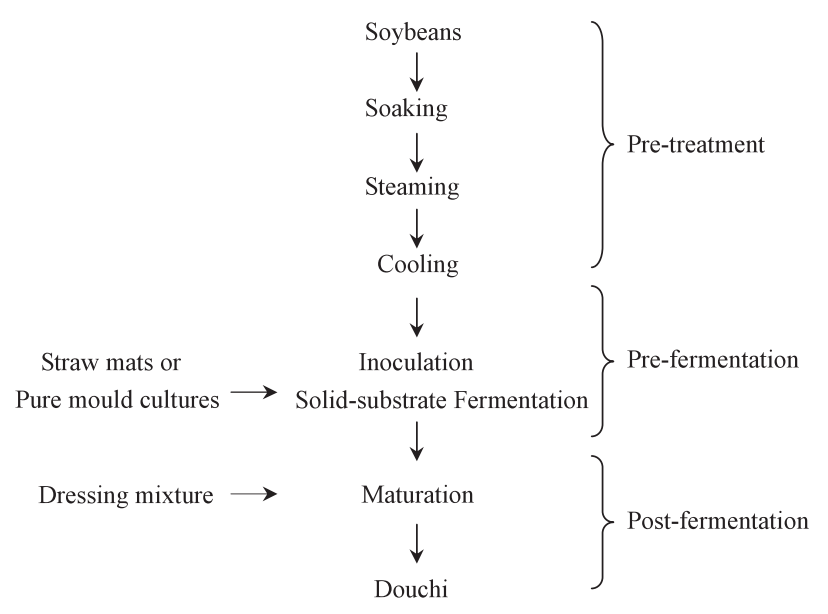

Fig. 1. The schematic diagram for production of douchi

douchi.

Isoflavones are a kind of flavonoid which have four chemical forms: the aglycones daidzein, genistein and glycitein; the glucosides daidzin, genistin and glycitin; the acetylglucosides 6"- $O$-acetyl-daidzin, 6"-O-acetyl-genistin and 6"-O-acetyl-glycintin; and the malonylglucosides 6"-O-Malonyl-daidzin, 6"-O-malonyl-genistin and 6"-Omalonyl-glycintin ${ }^{10}$.

Soybean isoflavones have been widely studied because of their physiological properties. They are known as phytoestrogens and have been shown to be effective in preventing osteoporosis ${ }^{8}$, lowering breast cancer risk ${ }^{12}$, inhibiting oxidative damage ${ }^{11}$, and alleviating menopausal symptoms $^{8}$. The effects depend on the structures of isoflavones in various bioactivities. It has been reported that isoflavone aglycones were more effective than glucosides in regulating cholesterol and fatty acids metabolism in rats ${ }^{9}$. Genistein was reported to have a higher anti-proliferative effect on the growth of human breast carcinomas and prostate cancer cells than genistin ${ }^{17,21}$. Several lines of evidence showed that daidzein and genistein are more bioavailable than the conjugated forms in rat stomachs ${ }^{22}$.

Owing to the specific structure, soy isoflavones can improve cognitive function in both humans and rats and prevent the degeneration of the central nervous system and the development of Alzheimer's disease (AD) ${ }^{5,19}$. It has been reported that acetylcholinesterase activity in the soy isoflavones diet fed animals was significantly inhibited in the cortex, basal forebrain and hippocampus compared with animals fed the control diet ${ }^{13}$. In a more recent study, soy isoflavones treatment could result in a significant decrease in acetylcholinesterase (AChE) activity and increase in the contents of some amino acid neurotransmitters such as glutamic and aspartic acids in the frontal cerebral cortex and hippocampus of mice ${ }^{14}$. In a different study, a soy diet that was rich in isoflavones was able to reverse the increase of $\mathrm{AChE}$ in hippocampus of ovariectomized rats $^{15}$. In addition, Pal and Tandon (1998) reported that genistein extracted from the root-tuber peel of Flemingia vestita could significantly inhibit the activity of AChE in Raillietina echinobothrida ${ }^{18}$. These findings show that soy isoflavones can influence the brain cholinergic system and reduce cognition decline in animal models.

In the present study, we investigated the content and composition of isoflavones in 19 samples of representative commercial douchi in China, as well as examining their AChE inhibitory activity in vitro for the first time. Moreover, the correlation between isoflavone content and $\mathrm{AChE}$ was also discussed. The purpose was to estimate the healthful effects of Chinese douchi in relation to isoflavones content and a potential AD-preventive agent derived from this food.

\section{Materials and methods}

\section{Materials}

Nineteen representative samples of douchi were obtained from local markets in different regions in China (Table 1). The types of douchi are shown according to the previous literature ${ }^{2,29}$. Acetylcholinesterase from human recombinant $(2,220 \mathrm{U} / \mathrm{mg}$ protein, E.C. No. 3.1.1.7), acetylthiocholine iodide (ATChI), and 5,5'-dithiobis[2nitrobenzoic acid] (DTNB) were purchased from Sigma Chemical Co. (St. Louis, MO). Galanthamine hydrobromide was obtained from NICPBP (National Institute for the Control of Pharmaceutical and Biological Products, Beijing, China). All other chemicals were of analytical grade.

Daidzin, genistin, glycitin, daidzein, genistein, and glycitein were purchased from Sigma Chemical Co. (St. Louis, MO). 6"-O-Malonyl-daidzin, 6"-O-malonyl-genistin, 6"-O-malonyl-glycintin, 6"-O-acetyl-daidzin, 6"-Oacetyl-genistin, and 6"-O-acetyl-glycintin were purchased from Wako Pure Chemical Co., Ltd. (Osaka, Japan).

\section{Preparation of douchi extracts}

The douchi samples were freeze-dried and pulverized into powder using a mortar and a pestle, and stored in brown glass bottles at $-20^{\circ} \mathrm{C}$ until used.

One gram of lyophilized douchi powder was suspended in $10 \mathrm{~mL} 80 \%(\mathrm{v} / \mathrm{v})$ ethanol. The mixture was then homogenized using a T25BS4 homogenizer (IKA Labortechnik, Staufen, Germany). The homogenized samples were extracted for $20 \mathrm{~min}$ in an ultrasonic water bath. Then the mixture was centrifuged at $3,000 \times \mathrm{g}$ for $15 \mathrm{~min}$ at $4^{\circ} \mathrm{C}$, and then the supernatant was filtered through a 0.45 $\mu \mathrm{m}$ syringe filter unit (Millex-HX, Millipore, USA). The filtrate was diluted by phosphate buffer $(0.1 \mathrm{M}, \mathrm{pH} 8.04)$ 
Table 1. Brand names, origins, type of soybean used and types of commercial douchi samples

\begin{tabular}{cllll}
\hline \hline Sample code & Brands & \multicolumn{1}{c}{ Origins } & Type of soybean & Types of douchi \\
\hline 1 & C.W & Sichuan (mid-west) & Yellow & Mucor-type \\
2 & T.C & Sichuan (mid-west) & Black & Mucor-type \\
3 & R.M & Chongqing (mid-west) & Yellow & Mucor-type \\
4 & Y.C & Chongqing (mid-west) & Yellow & Mucor-type \\
5 & Q.M & Chongqing (mid-west) & Yellow & Mucor-type \\
6 & A.J & Chongqing (mid-west) & Yellow & Mucor-type \\
7 & Y.F & Guangdong (south) & Black & Aspergillus-type \\
8 & X.Q & Guangdong (south) & Black & Aspergillus-type \\
9 & Y.J.Q & Guangdong (south) & Black & Aspergillus-type \\
10 & Y.M & Guangxi (south) & Black & NC \\
11 & Q.X & Guizhou (central) & Yellow & Bacillus-type \\
12 & K.M & Yunnan (south-west)) & Yellow & Bacillus-type \\
13 & T.M.S & Hunan (central) & Black & Aspergillus-type \\
14 & T.P.Q & Hunan (central) & Black & Aspergillus-type \\
15 & L.Y..P & Hunan (central) & Black & Aspergillus-type \\
16 & Q.W & Hunan (central) & Black & Aspergillus-type \\
17 & D.X.Y & Jiangxi (mid-south) & Black & Aspergillus-type \\
18 & K.W.K & Shanghai (east) & Black & Aspergillus-type \\
19 & Y.B & Jilin (north-east) & Yellow & NC \\
\hline
\end{tabular}

NC: not clear.

to appropriate concentration for further AChE inhibitory activity assay.

\section{Determination of isoflavones contents of douchi extracts}

The contents of isoflavones in the douchi extracts were determined as described previously ${ }^{31}$. Freeze-dried powder $(2 \mathrm{~g})$ was extracted with $50 \mathrm{ml}$ of $80 \%(\mathrm{v} / \mathrm{v})$ methanol at $80^{\circ} \mathrm{C}$ for $4 \mathrm{~h}$ by a Soxhlet extractor and the extractants filtered through a $0.45 \mu \mathrm{m}$ filter unit. Isoflavones were analyzed quantitatively by high pressure liquid chromatography (HPLC). The HPLC system used was a Shimadzu HPLC (Kyoto, Japan), consisting of an LC10AT pump, a UV detector (SPD-10AVVP), and a Dikma Diamonsil $\mathrm{C}_{18}$ column $(4.6 \times 250 \mathrm{~mm}$, Dima Co., Ltd., Orlando, FL). The mobile phases for HPLC consisted of solvent (A) $0.1 \%(\mathrm{v} / \mathrm{v})$ acetic acid in filtered Milli-Q water, and (B) $0.1 \%(\mathrm{v} / \mathrm{v})$ acetic acid in acetonitrile. The solvent gradient was as follows: Solvent B was increased from 15 to $25 \%$ over $35 \mathrm{~min}$, then increased to $26.5 \%$ within the next $12 \mathrm{~min}$, and finally increased to $50 \%$ within $30 \mathrm{~s}$ prior to being held for $14.5 \mathrm{~min}$. The flow rate was $1.0 \mathrm{~mL} / \mathrm{min}$. The column temperature was $40^{\circ} \mathrm{C}$ and the absorption was measured at $254 \mathrm{~nm}$. Quantitative data for each isoflavone was obtained by comparison to known standards. In order to estimate total isoflavone amounts, individual isoflavone glucosides and aglycones were normalized for their molecular weight differences and summed, and regarded as total isoflavones.

\section{Determination of $\mathrm{AChE}$ inhibitory activity}

The AChE inhibitory activities of douchi extracts were determined according to Ellman's colorimetric method with some modifications ${ }^{4}$. In the 96-well plates, $150 \mu \mathrm{L}$ of phosphate buffer $(0.1 \mathrm{M}, \mathrm{pH} 8.04)$ was added to the wells followed by $30 \mu \mathrm{L}$ of sample solution, $50 \mu \mathrm{L}$ of DTNB $(756 \mu \mathrm{M})$ and $20 \mu \mathrm{L}$ of AChE enzyme $(0.54$ $\mathrm{U} / \mathrm{mL})$. The mixture was incubated for $5 \mathrm{~min}$ at $37^{\circ} \mathrm{C}$. Following pre-incubation, $50 \mu \mathrm{L}$ of the substrate (ATChI, $3 \mathrm{mM}$ ) was added in the above mixture solution. The absorbance of the resulting solution was measured at 405 $\mathrm{nm}$ every $28 \mathrm{~s}$ for ten times using a 96-well plate reader (Model 680, Bio-Rad Laboratories, Tokyo, Japan). Absorbance was plotted against time and enzyme activity was calculated from the slope of the line so obtained and expressed as a percentage compared with an assay using a buffer without any inhibitor. The percent inhibition was calculated by the following formula.

$$
\operatorname{inhibition}(\%)=\frac{K_{\text {conrol }}-K_{\text {sample }}}{K_{\text {control }}-K_{\text {negative }}} \times 100
$$

The AChE inhibition activities were expressed as $\mathrm{IC}_{50}$, which is the inhibitory concentration of the test samples that inhibits $50 \%$ AChE by log-probit analysis. Galanthamine hydrobromide was used to benchmark the AChE inhibitory activity. Thirty $\mu \mathrm{L}$ of $10 \%$ ethanol in buffer was used as the negative control instead of the douchi extract. Tests were carried out in triplicate. 


\section{Statistical analysis}

The mean values of three experiments were calculated. The SAS system (SAS for Windows 6.12, SAS Institute Inc., Cary, NC, USA) was used for statistical analysis. Duncan's multiple range tests were used to estimate significant differences among the mean values at the $5 \%$ probability level.

\section{Results and discussion}

\section{Isoflavone content and composition of douchi extracts}

Nineteen brands of douchi samples collected from various parts of China were investigated. The samples can be regarded as representative of the major types of douchi found in China. The brand names, origins, species types of soybean and microorganism species used for douchi preparation are shown in Table 1.

Isoflavone contents of douchi extracts are shown in Table 2. The total content of isoflavones in 19 brands of douchi samples ranged from 24 to $1,471 \mu \mathrm{g} / \mathrm{g}$ dry matter. Similar distribution patterns of isoflavones were observed among the 19 brands of douchi samples. Isoflavone aglycones accounted for $75 \%$ to $96 \%$ of total isoflavones contents (except No. 6 and No. 19). Daidzein and genistein were the major isoflavone constituents in douchi samples. The content of malonylglucosides was the lowest in isoflavone profiles. 6"-O-Acetylglycitin was not detected in any of the samples in the experiment. These results were consistent with previous literature ${ }^{6,27,31}$.

Isoflavone content and distribution in soy-based foods were reported to depend on the variety of soybean, methods of processing, and addition of other components. Wang and Murphy evaluated the concentration and distribution of isoflavones in 29 commercial soybean foods ${ }^{27}$. Traditional soybean foods showed differences between non-fermented and fermented food. Non-fermented foods had greater levels of glucosides, while in contrast, greater levels of aglycones were found in fermented foods.

Processing techniques, such as soaking, heat treatment and fermentation, not only influenced the content of isoflavones but also changed the forms of isoflavones ${ }^{7,31,33}$. During soaking, the concentration of daidzin-genistin decreases accordingly to the daidzein-genistein formation ${ }^{24,32}$. It was reported that the malonylated isoflavone glucosides were thermally unstable, and were converted into their corresponding isoflavone glycosides and aglycones at the higher temperature ${ }^{3,28}$. In many respects, isoflavones convert mainly from glucosides to aglycones under the $\beta$-glucosidases from microbial source hydrolysis in the fermentation process ${ }^{23,31}$.
From Table 2, the average content of isoflavone aglycones of Aspergillus-type douchi was two times higher than that of Mucor-type or Bacillus-type douchi, which had average contents of $857 \mu \mathrm{g} / \mathrm{g}, 384 \mu \mathrm{g} / \mathrm{g}$ and $380 \mu \mathrm{g} / \mathrm{g}$, respectively. Although this might be relevant to the variety of soybean, the microorganism species and processing methods in douchi production may be the main factor of the difference. The different microorganism and inocula would provide different extents of hydrolysis activities during the fermentation. Moreover, in contrast with Aspergillus-type douchi, Mucor-type douchi was made during winter and had a year long fermentation time. Since the appropriate temperature of $\beta$-glucosidase was generally higher than $30^{\circ} \mathrm{C}$, the fermentation season may affect the content of isoflavone aglycones. Meanwhile, fermentation time and non-soy ingredients might also influence the isoflavones contents.

The total contents of daidzein, glystein and genistein varied extremely in douchi samples which were made by the same microorganism. Compared with other fermented soybean food, such as tempeh $(625 \mu \mathrm{g} / \mathrm{g})^{27}$ and miso (895 $\mu \mathrm{g} / \mathrm{g})^{34}$, Chinese douchi has higher isoflavone contents especially Aspergillus-type douchi which ranged from 636 $\mu \mathrm{g} / \mathrm{g}$ to $1,471 \mu \mathrm{g} / \mathrm{g}$. Therefore, Chinese douchi is a better soybean food for consuming dietary isoflavone in a daily diet.

\section{The AChE inhibitory activity of douchi extracts}

In this study, the AChE inhibitory activities of 19 brands of Chinese douchi samples were examined. From Fig. 2., the $\mathrm{IC}_{50}$ values of douchi extracts ranged from 0.040 to $2.319 \mathrm{mg} / \mathrm{mL}$. Among these samples, some showed high inhibitory activities (No. 18, $0.040 \mathrm{mg} / \mathrm{mL}$ and No. 2, $0.041 \mathrm{mg} / \mathrm{mL}$ ), and some samples showed low inhibitory activities (No. 6, $2.319 \mathrm{mg} / \mathrm{mL}$ and No. 11, $0.992 \mathrm{mg} / \mathrm{mL}$ respectively).

Compared with the medicinal plants, douchi extracts had the better AChE inhibitory activity. Mukherjee reported the AChE inhibitory activity of six plant materials which are commonly used to improve memory function traditionally as central nervous system active plants in the Indian system of medicine. The $\mathrm{IC}_{50}$ values ranged from $106.55 \mu \mathrm{g} / \mathrm{mL}$ to $222.41 \mu \mathrm{g} / \mathrm{mL}^{16}$. A similar research found that the $\mathrm{IC}_{50}$ values of several plants of the Amaryllidaceae family ranged from $16.74 \mu \mathrm{g} / \mathrm{mL}$ to 73.69 $\mu \mathrm{g} / \mathrm{mL}^{25}$. This indicated that douchi can be considered as a new potential specific food in preventing the onset of human AD.

In addition, the samples from the same region showed similar inhibitory activity (No. 7, No. 8 and No. 9). The samples from Sichuan and Chongqing showed low inhibitory activity (No. 1 and No. 6). Although the AChE in- 


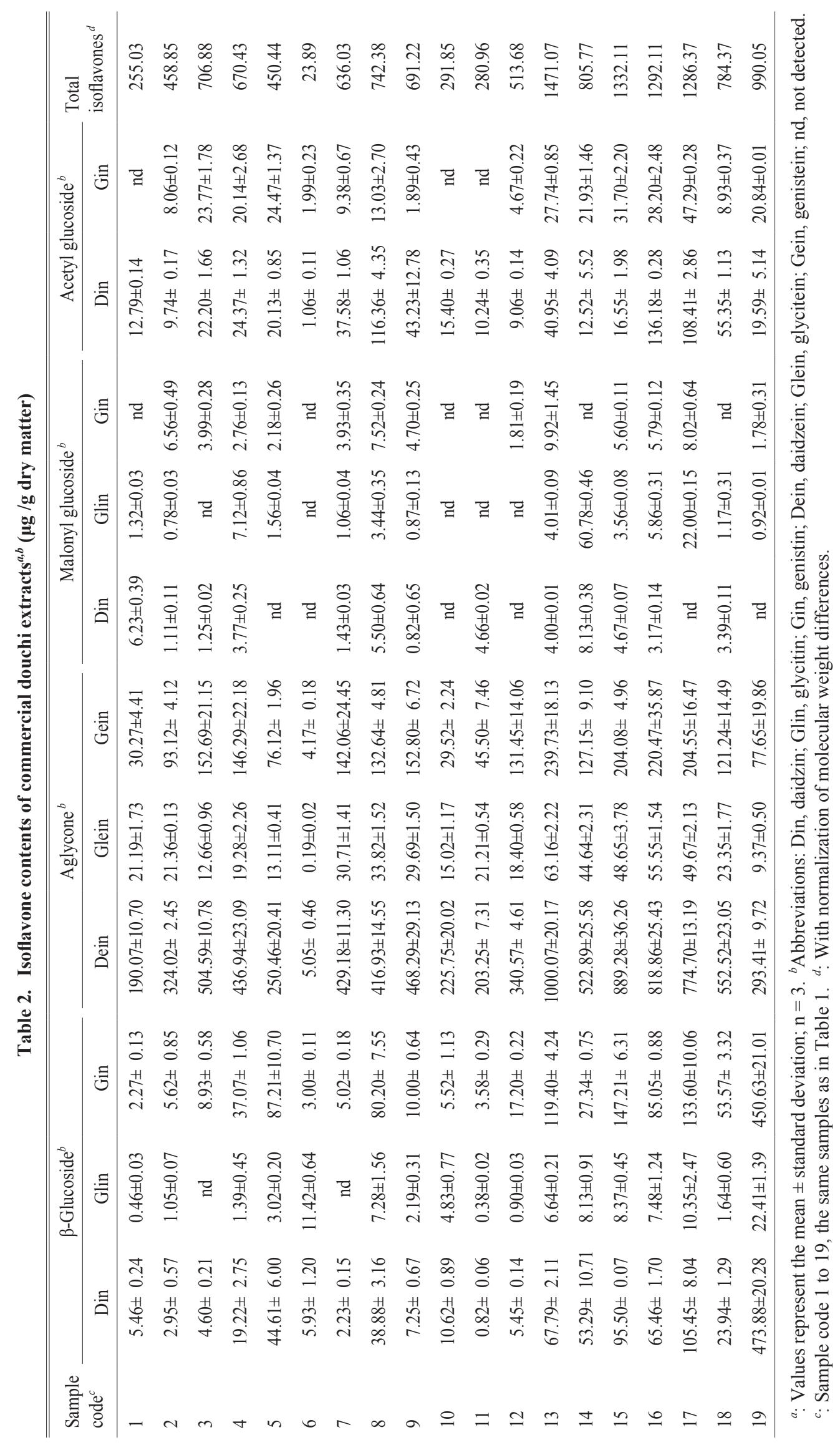




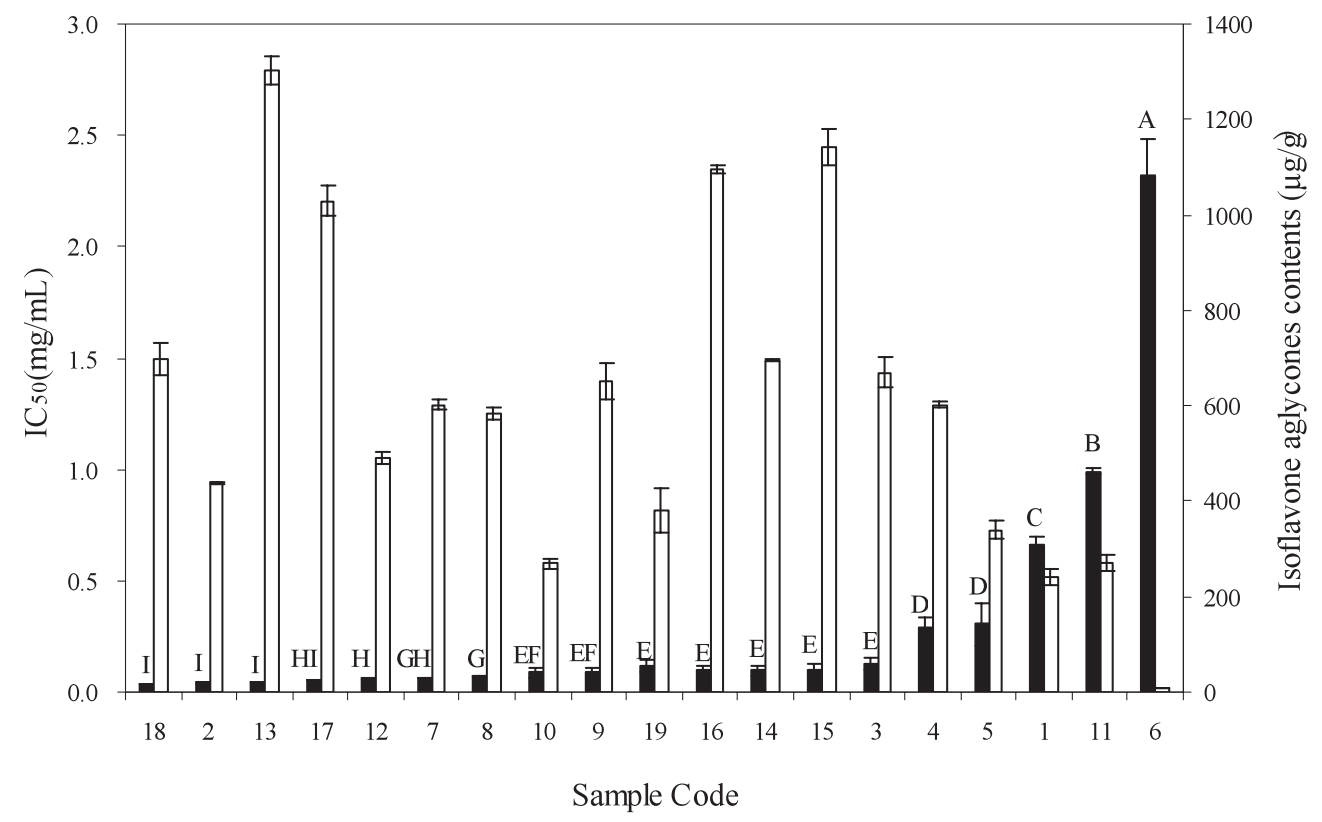

Fig. 2. Isoflavone aglycones contents and the $\mathrm{AChE}$ inhibitory activities of various douchi extracts Values are means of 3 replications. Vertical bars indicate standard errors.

Sample codes 1 to 19 are the same samples as in Table 1. Samples are rearranged in order of $\mathrm{IC}_{50}$ values. $\mathrm{IC}_{50}$ : concentration of crude extract required to inhibit enzyme activity by $50 \%$. Means for $\mathrm{IC}_{50}$ without a common letter are significantly different at $p<0.05$.

$\square$ : Isoflavone aglycones contents, $\square$ : $\mathrm{IC}_{50}$.

hibitory activities of the other douchi samples's extracts were also influenced by producing region, there was no clear directional tendency between the region of production and high AChE inhibitory activity.

From this study, significant variations in $\mathrm{AChE}$ inhibitory activity of extracts from different types of douchi were also observed. Four brands of low AChE inhibitory activity (samples No. 1 , No. 4, No. 5, and No. 6) all belonged to Mucor-type douchi, while almost all Aspergillus-type douchi extracts had significantly higher AChE inhibitory activity than that of Mucor-type and Bacillustype douchi. Their average $\mathrm{IC}_{50}$ values were $0.074 \mathrm{mg} / \mathrm{mL}$ (Aspergillus-type), $0.623 \mathrm{mg} / \mathrm{mL}$ (Mucor-type) and 0.526 $\mathrm{mg} / \mathrm{mL}$ (Bacillus-type), respectively. In the experiment, we also discovered that some brands of douchi (samples No. 1, No. 11 and No. 6) exhibited high $\mathrm{IC}_{50}$ values and low contents of isoflavone aglycones at the same time. However, high inhibition activities did not mean high isoflavone content, maybe some other substances contributed to the inhibition. Further studies to validate and determine the effect of isoflavone on AChE inhibitory activity, as well as other substances in douchi that may have an effect on inhibiting $\mathrm{AChE}$ should be considered.

\section{Conclusions}

In this study, the concentration and distribution of isoflavones and $\mathrm{AChE}$ inhibitory activity in 19 Chinese commercial douchi were evaluated. As a traditional fermented soybean food, aglycones were the main forms of isoflavones in Chinese commercial douchi. The total content of isoflavones varied significantly between the brands of douchi products. Aspergillus-type douchi is superior to the Mucor-type and Bacillus-type douchi in the isoflavones content. Furthermore, Chinese douchi showed different extents of $\mathrm{AChE}$ inhibitory activities, and the $\mathrm{IC}_{50}$ values ranged from 0.040 to $2.319 \mathrm{mg} / \mathrm{mL}$. Some of the douchi samples showed strong inhibitory effects on $\mathrm{AChE}$ with $\mathrm{IC}_{50}$ values of 0.040 and $0.041 \mathrm{mg} / \mathrm{mL}$. Aspergillus-type douchi exhibited significantly higher AChE inhibitory activity than that of Mucor-type and Bacillus-type douchi. Some brands of douchi which have a low content of isoflavone aglycones showed much lower inhibitory activity. However, high inhibition activities did not mean high isoflavone content, maybe some other substances contributed to the inhibition.

\section{Acknowledgments}

This study was conducted within the framework of the collaborative research project between Japan and China titled "Development of sustainable production and utilization of major food resources in China" supported by Japan International Research Center for Agricultural Sciences 
(JIRCAS). This work was also supported by National Key Technologies R\&D Program (National Functional Food Project) in the Eleventh Five-year Plan of the People's Republic of China (No.2006BAD27B09).

\section{References}

1. Bao, Q. A. (1985) The headstream and technology of Douchi. China Brewing, 2, 9-14.

2. Chen, J. et al. (2007) Anti- $\alpha$-glucosidase activity of Chinese traditionally fermented soybean (douchi). Food Chem., 103, 1091-1096.

3. Chiarello, M. D. et al. (2006) Influence of heat treatment and grain germination on the isoflavone profile of soy milk. J. Food Biochem., 30, 234-247.

4. Ellman, G. L. et al. (1961) A new and rapid colorimeteric determination of acetylcholineesterase activity. Biochem. Pharmacol., 7, 88-95.

5. File, S. E. et al. (2001) Eating soya improves human memory. Psychopharmacology (Berl), 157, 430-436.

6. Fukutake, M. et al. (1996) Quantification of genistein and genistin in soybeans and soybean products. Food Chem. Toxicol., 34, 457-461.

7. Ha, E. Y. W., Morr, C. V. \& Seo, A. (1992) Isoflavone aglucones and volatile organic compounds in soybeans: Effects of soaking treatments. J. Food Sci., 57, 414-417.

8. Ishida, H., et al. (1998) Preventive effects of the plant isoflavones, daidzin and genistin on bone loss in ovariectomized rats fed a calcium-deficient diet. Biol. Pharm. Bull., 21, 62-66.

9. Kawakami, Y. et al. (2005) Comparison of regulative functions between dietary soy isoflavones aglycone and glucoside on lipid metabolism in rats fed cholesterol. J. Nutr. Biochem., 16, 205-212.

10. Kudou, S. et al. (1991) Malonyl isoflavone glycosides in soybean seeds (Glycine max MERRILL). Agric. Biol. Chem., 55, 2227-2233.

11. Lai, H. H. \& Yen, G. C. (2002) Inhibitory effect of isoflavones on peroxynitritemediated low-density lipoprotein oxidation. Biosci. Biotechnol. Biochem., 66, 22-28.

12. Lee, H. P. et al. (1991) Dietary effects on breast-cancer risk in Singapore. Lancet, 337, 1197-1200.

13. Lee, Y. B. et al. (2004) Soy isoflavones improve spatial delayed matching-to-place performance and reduce cholinergic neuron loss in elderly male rats. J. Nutr., 134, 1827-1831.

14. Liu, Y. Q. et al. (2007) Memory performance of hypercholesterolemic mice in response to treatment with soy isoflavones. J. Neurosci. Res., 57, 544-549.

15. Monteiro, S. C. et al. (2007) Supplementation with vitamins $\mathrm{E}$ plus $\mathrm{C}$ or soy isoflavones in ovariectomized rats: effect on the activities of $\mathrm{Na}^{+}, \mathrm{K}^{+}$-ATPase and cholinesterases. Metab. Brain Dis., 22, 156-171(16).

16. Mukherjee, P. K., Kumar, V. \& Houghton, P. J. (2007) Screening of Indian medicinal plants for acetylcholinesterase inhibitory activity. Phytother. Res., 12, 1142-1145.

17. Onozawa, M. et al. (1998) Effects of soybean isoflavones on cell growth and apoptosis of the human prostatic cancer cell line LNCaP. Jpn. J. Clin. Oncol., 28, 360-363.

18. Pal, P. \& Tandon, V. (1998) Anthelmintic efficacy of Flemingia vestita (Fabaceae): Genistein-induced alterations in the esterase activity in the cestode, Raillietina echinobothrida. J. Biosci., 23, 25-31.

19. Pan, Y. et al. (2000) Soy phytoestrogens improve radial arm maze performance in ovariectomized retired breeder rats and do not attenuate benefits of $17 \beta$-estradiol treatment. Menopause, 7, 230-235.

20. Peng, Y. et al. (2003) Purification and characterization of a fibrinolytic enzyme produced by Bacillus amyloliquefaciens DC-4 screened from douchi, a traditional Chinese soybean food. Comp. Biochem. Physiol. B Biochem. Mol. Biol., 134, 45-52.

21. Peterson, G. \& Barnes, S. (1991) Genistein inhibition of the growth of human breast cancer cells: independence from estrogen receptors and the multi-drug resistance gene. Biochem. Biophys. Res. Comm., 179, 661-667.

22. Piskula, M. K., Yamakoshi, J. \& Iwai, Y. (1999) Daidzein and genistein but not their glucosides are absorbed from the rat stomach. FEBS Lett., 447, 287-291.

23. Ribeiro, M. L. L. et al. (2006) $\beta$-glucosidase activity and isoflavone content in germinated soybean radicles and cotyledons. J. Food Biochem., 30, 453-465.

24. Toda, T. et al. (2001) Changes in isoflavone compositions of soybean during soaking in water. Food Sci. Technol. Res., 7, 171-175.

25. Vinutha, B. et al. (2007) Screening of selected Indian medicinal plants for acetylcholinesterase inhibitory activity. J. Ethnopharmacol., 109, 359-363.

26. Wang, D. et al. (2008) In vitro and in vivo studies on the antioxidant activities of the aqueous extracts of Douchi (a traditional Chinese salt-fermented soybean food). Food Chem., 107, 1421-1428.

27. Wang, H. J. \& Murphy, P. A. (1994) Isoflavone content in commercial soybean foods. J. Agric. Food Chem., 42, 1666-1673.

28. Wang, H. J. \& Murphy, P. A. (1996) Mass balance study of isoflavones during soybean processing. J. Agric. Food Chem., 44, 2377-2383.

29. Wang, L. J. (2006) Studies on the fermentation mechanism and volatile components of Aspergillus-type douchi. Ph. D. thesis, China Agricultural University, Beijing, China, pp. 2 [In Chinese].

30. Wang, L. J. et al. (2007) Antioxidative activity of Douchi (a Chinese traditional salt-fermented soybean food) extracts during its processing. Int. J. Food Prop., 10, 385-396.

31. Wang, L. J. et al. (2007) Influences of processing and $\mathrm{NaCl}$ supplementation on isoflavone contents and composition during Douchi manufacturing. Food Chem., 101, 12471253.

32. Wardhani, D. H., Vázquez, J. A. \& Pandiella, S. S. (2008) Kinetics of daidzin and genistin transformations and water absorption during soybean soaking at different temperatures. Food Chem., 111, 13-19.

33. Xu, Z., Wu, Q. \& Godber, J. S. (2002) Stabilities of daidzin, glycitin, genistin, and generation of derivatives during heating. J. Agric. Food Chem., 50, 7402-7406.

34. Yamabe, S. et al. (2007) Effect of soybean varieties on the content and composition of isoflavone in rice-koji miso. Food Chem., 100, 369-374.

35. Zhang, J. H. et al. (2006) Angiotensin I-converting enzyme inhibitory peptides in douchi, a Chinese traditional fermented soybean product. Food Chem., 98, 551-557. 